\title{
Simulation and prediction of uptake, distribution, and exhalation of organic solvents
}

\author{
VERA FISEROVA-BERGEROVA', JIRI VLACH ${ }^{2}$, AND \\ KISHORE SINGHAL \\ Department of Anesthesiology, University of Miami School of Medicine, Miami, Florida, USA' \\ and Department of Electrical Engineering, University of Waterloo, Waterloo, Ontario, Canada
}

\begin{abstract}
Fiserova-Bergerova, Vera, Vlach, J., and Singhal, K. (1974). British Journal of Industrial Medicine, 31, 45-52. Simulation and prediction of uptake, distribution, and exhalation of organic solvents. Presented here is a theory and mathematical solution of the uptake, distribution, and excretion of inhaled lipid soluble noxious gases, which embraces the effects of metabolism. An electrical analogue was employed to explain the theory, since the analogue is described by the same set of differential equations, and much knowledge is available for the mathematical treatment of electrical networks. The model is used to calculate the uptake and exhalation curves of vapours, to explain what happens to the inhaled vapours in the body, and to predict their cumulation in the body in periodic situations such as occur in industrial exposure.
\end{abstract}

It was established experimentally that the kinetics of the uptake and excretion of drugs are governed by exponential functions. A theoretical background is available for the uptake and excretion of anaesthetics (Uptake and Distribution of Anesthetic Agents, 1963). However, the theory is valid only for inert gases, as it does not take into account the effects of metabolism. This paper attempts to fill this theoretical gap and to demonstrate the significant role played by metabolism in the uptake, distribution, and excretion of lipid soluble vapours.

The pharmacokinetics of inhaled compounds are determined by two factors: (1) the transport rate of the compound from lung to tissue and from tissue back to lung; and (2) the capacity of the tissue to retain the compound.

Transport rate and tissue absorption capacity vary with different tissues (Eger, 1963). The whole body uptake for a given compound is the sum of the uptakes of the various physiological compartments.

The rate constants ' $k$ ' or their reciprocal time constants ' $\tau$ ' in the exponents can be predicted from the solubility of the tested compound (partition coefficient $\lambda$ tissue/blood) and from certain physiological factors (blood perfusion of tissue ' $F$ ' and tissue volume ' $V$ ') by the equation:

$$
\begin{aligned}
& \frac{1}{\tau}=k=\frac{\text { transportation rate }}{\text { tissue capacity }}=\frac{c_{\text {in }} \times F \times \lambda_{\mathrm{bl}} / \mathrm{air}}{c_{\text {in }} \times V \times \lambda_{\mathrm{tis}} / \mathrm{air}} \\
& \frac{F}{V \times \lambda_{\mathrm{tis}} / \mathrm{bl}}
\end{aligned}
$$

As can be seen from this equation, the concentration of noxious vapour in the air during the exposure, that is, $c_{\text {in }}$, has no effect on the rate constant.

If the tissues are grouped according to similar perfusion rates and according to similar partition coefficients tissue/blood, three distinctly recognizable pharmacokinetic compartments are apparent (Eger, 1963): (1) the tissue of the vessel-rich group (VRG); (2) low perfused tissue such as muscle and skin (MG); and (3) poorly perfused fatty tissue with a large partition coefficient (FG). The time constants of the first two compartments for lipid soluble compounds 
are in the range of minutes, whereas the time constants of the third compartment are in the range of several hours.

Pulmonary uptake during a single exposure can be fairly easily explained, since the initial conditions (the amount of drug in the body) are zero. The desaturation process is more difficult to explain as the initial conditions are non-zero and depend on the duration of exposure. During saturation the drug is redistributed among the various compartments.

The mutual influences of the compartments can be modelled by an electrical network containing resistors and capacitors (Severinghaus, 1963; Mapleson, 1963a, 1963b; Fiserova-Bergerova and Cettl, 1972). The explanation, simulation, and prediction of the uptake and excretion of drugs is then simplified as considerable knowledge is available from the field of electrical engineering for the analysis of such networks. The transport of inhaled drugs from the lung to the three pharmacokinetic compartments can be simulated by four pairs of properly interconnected resistors and capacitors. Uptake and excretion are simulated by charging and discharging such a network.

In the pharmacokinetic studies done so far on anaesthetics, the effects of metabolism have been neglected, even though metabolism represents a significant body output of drugs. A large part of metabolism takes place in the liver, but approximately 10 to $20 \%$ of microsomal enzyme activity takes place in other organs (Conney, 1972). Cytochrome P-450 has been found in tissues which are included in the VRG compartment. Teisinger and Soucek (1952) have shown that the metabolic rate of an inhaled substance is proportional to the inhaled concentration and that the factor is the metabolic coefficient. The metabolic coefficient is easy to determine from the uptake rate measured after a sufficiently long period of exposure when the viscera and muscles are near saturation. At this stage the total uptake is the sum of the uptake by adipose tissue (predictable from solubility) and the metabolic output. The metabolic coefficient $K_{M}$ is defined as the difference between the measured uptake rate $\left(a_{m}\right)$ and the uptake rate predicted by drug solubility $\left(a_{p}\right)$ divided by the concentration of drug in inhaled air $\left(c_{\text {in }}\right)$ (Teisinger and Soucek, 1952).

$$
K_{M}=\frac{a_{m}-a_{p}}{c_{\text {in }}}
$$

The metabolic coefficient has been taken into account in the modelling and computation done on the analogue computer (Fiserova-Bergerova and Cettl, 1972).

The purposes of this study are the following:

1. simulation of uptake and excretion by an electrical network, determining the values of the resistors and capacitors in the network from phy- siological and physical data for inhaled lipid soluble metabolized compounds;

2. application of mathematical procedures valid for chronic and long-term exposures;

3. demonstration that simulation by an electrical network is a powerful tool for prediction;

4. demonstration of the effects of metabolism on the distribution of noxious vapours in the body.

Benzene was the noxious compound chosen for the study to illustrate the correspondence between the measured and the calculated data.

\section{Experimental}

Uptake, distribution, and exhalation of drugs by three pharmacokinetic compartments were modelled by a network with different resistance-capacitance combinations (Fig. 1). As the drugs enter the different compartments through the channel representing the blood stream, the first resistor and capacitor $\left(R_{1}, C_{1}\right)$ account for pulmonary ventilation $\left(R_{1}\right)$ and functional residual capacity, lung tissue, and arterial blood (determining $C_{1}$ ). To calculate $R_{1}$, the total or alveolar ventilation was employed, according to whether vapour concentrations in inhaled and mixed exhaled air or in alveolar air were followed. The three pharmacokinetic compartments are 'connected' to the first R-C combination as other resistor-capacitor combinations. The capacitors are proportional to the capacities of these three compartments to absorb the particular drug, and the resistances are

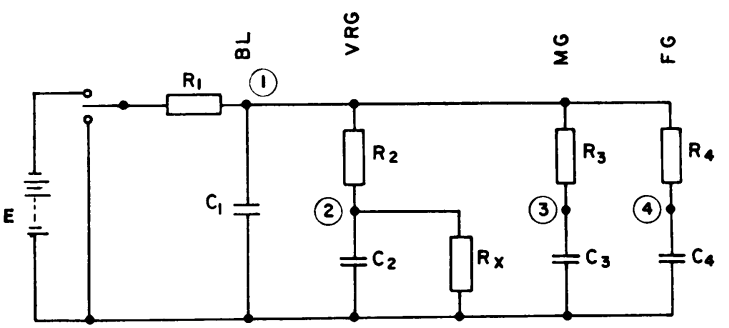

fIG. 1. Electrical network simulating the uptake, metabolism, and exhalation of volatile lipid soluble compounds.

$\mathrm{E}$ is the battery, Cs are the capacities representing the capacities of compartments to retain drug, and Rs are the resistors representing the drug transportation rates to individual pharmacokinetic compartments.

$\mathrm{R}_{1}=\frac{1}{\text { pulmonary ventilation }}$

$\mathrm{C}_{1}=$ FRC + lung tissue volume $\times \lambda$ tis/air + volume of arterial blood $\times \lambda$ blood/air.

$\mathrm{C}_{2}, \mathrm{C}_{3}, \mathrm{C}_{4}=$ tissue volume of individual pharmacokinetic compartment $\times \lambda$ tis/air.

$\frac{1}{R}, \frac{1}{R}, \frac{1}{R_{4}}=$ blood perfusion of the individual pharma$\overline{\mathbf{R}_{2}}, \overline{\mathbf{R}_{3}}, \overline{\mathbf{R}_{4}}=$ cokinetic compartment $\times \lambda$ blood/air.

$\mathbf{R}_{\mathbf{x}}=\frac{1}{\text { metabolic coefficient }}-\mathbf{R}_{\mathbf{1}}-\mathbf{R}_{\mathbf{2}}$. 
proportional to the reciprocals of the transport rates to the tissue.

In order to account for metabolism, as well as for the excretion of the unmetabolized drug, two outputs are provided for the discharge of the simulating electrical model: (1) Metabolism takes away some portion of the drug from the VRG group, which is expressed by the resistor $R_{x}$ in Figure 1. (2) Pulmonary excretion of the unmetabolized drug is simulated by discharge through the resistor $R_{1}$ after exposure has terminated (switch in lower position). Since the metabolic coefficient includes the chemical $\left(R_{x}\right)$ as well as the physical factors $\left(R_{1}, R_{2}\right)$ affecting metabolism, the value of $R_{x}$ is determined by the equation

$$
\mathbf{R}_{\mathbf{x}}=\frac{1}{K_{M}}-\mathbf{R}_{1}-\mathbf{R}_{\mathbf{2}}
$$

$\left(R_{1}\right.$ and $R_{2}$ are the reciprocal of the transportation rate of drugs to the metabolic site).

The data for tissue volume and blood perfusion of pharmacokinetic compartments of a standard man are the same as published by Eger (1963). Cardiac output $(\mathrm{Qv})$ was calculated from alveolar ventilation using the coefficient 1.25. The excess of blood flow (compared to basal cardiac output $Q$ ) is considered to perfuse the muscle group compartment (MG). The data for a standard man (cardiac output $6 \mathrm{l} / \mathrm{min}$ ) are in the Table. The partition co-efficients for benzene are taken from Kozáková (1955): blood/air 6; tissue/air 7; fat/air 400. The metabolic coefficient $\left(K_{M}\right)$ was estimated at $3 \cdot 2$ (Teisinger and Soucek, 1952). The mathematical solution is in the Appendix.

\section{TA B LE}

Pharmacokinetic Compartments for a Standard Man (Eger, 1963), $70 \mathrm{~kg}, 1.73 \mathrm{~m}^{2}$, Cardiac Output $6 \mathrm{l} / \mathrm{min}$

\begin{tabular}{l|c|ccc}
\hline & \multirow{2}{*}{ Unit } & \multicolumn{4}{|c}{ Compartment } \\
& & $V R G$ & $M G$ & $F G$ \\
\hline Volume & 1 & 6 & 33 & $14 \cdot 5$ \\
Blood perfusion & $1 /$ min & $4 \cdot 5$ & $1 \cdot 1$ & $0 \cdot 3$ \\
Volume/blood flow & min & $1 \cdot 3$ & 30 & 48 \\
\hline
\end{tabular}

VRG = liver, heart, kidney, brain, endocrine glands, and spinal cord

MG = muscle and skin

FG = fat and fatty marrow

$\mathrm{FRC}=2.71$.

Volume of lung tissue $V=0.61$.

Volume of arterial blood $V_{\mathrm{bl}}=1.41$.

\section{Results}

Experiments were performed with 14 volunteers exposed continuously for five hours to a constant concentration of benzene (Teisinger, FiserovaBergerova, and Kudrna, 1952; Teisinger and FiserovaBergerova, 1955). The data for a standard man, ventilation $8 \mathrm{l} / \mathrm{min}$, were used for computation. The

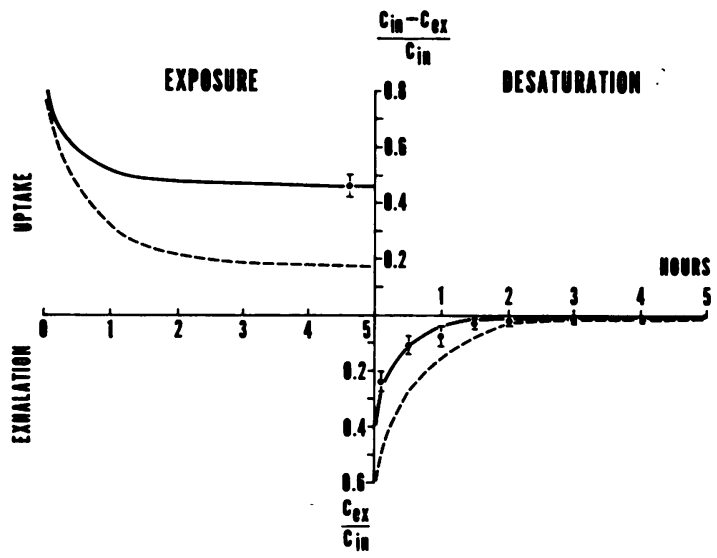

FIG. 2. Pulmonary uptake and excretion of benzene following five hours of exposure.

Abscissa represents time after the beginning of exposure or of desaturation respectively, in hours; ordinate represents uptake $\left(\mathrm{c}_{\mathrm{in}}-\mathrm{c}_{\mathrm{ex}}\right)$ or exhalation $\left(\mathrm{c}_{\mathrm{ex}}\right)$ respectively, related to the concentration of benzene in the inhaled air during the exposure period $\left(\mathrm{c}_{\text {in }}\right)$.

Unbroken line is computed for $K_{M}=3.21 / \mathrm{min}$; broken line for $K_{M}=10^{-4} \mathrm{l} / \mathrm{min}$. Mean and standard error of the mean of measurements in 14 volunteers are included.

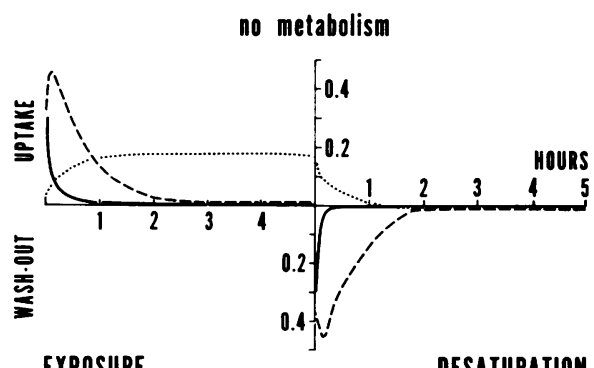

with metabolism

DESATURATION

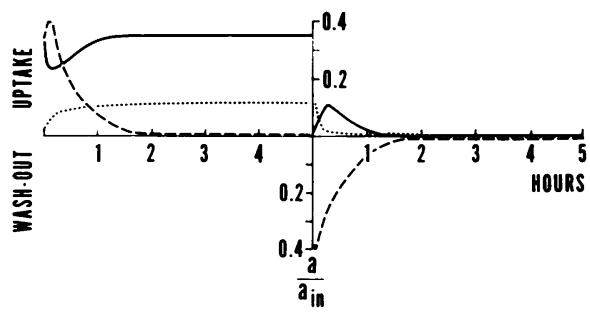

FIG. 3. Effects of metabolism on uptake and washout of benzene from pharmacokinetic compartments.

Ordinate represents uptake or washout of benzene, respectively ( $a$ in $\mathrm{mg} / \mathrm{min}$ ), related to the amount of benzene inhaled per minute of exposure $\left(a_{\text {in }}\right)$. Unbroken line (VRG), broken line (MG), and dotted line (FG). 
EXPOSURE
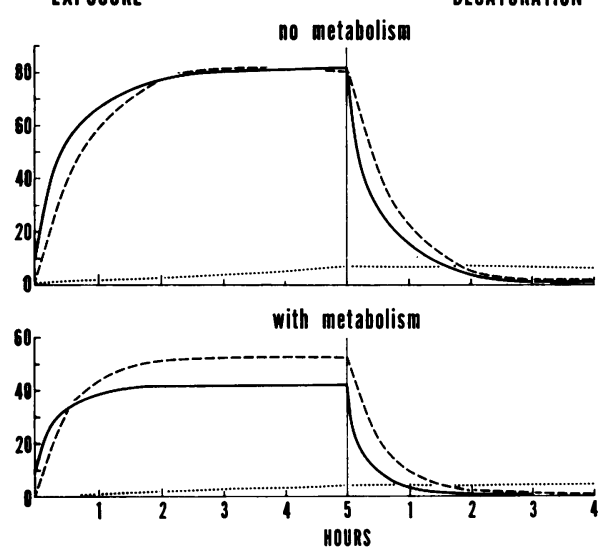

FIG. 4. Effect of metabolism on benzene levels in tissues of pharmacokinetic compartments.

Ordinate is percentage of saturation of the compartment, $\frac{\text { actual concentration in tissue } \times 100}{\text { concentration in inhaled air } \times \lambda \text { tis/air }}$. Unbroken line

(VRG), broken line (MG), and dotted line (FG).

experiment was simulated with $K_{M}$ equal to $3 \cdot 2$, as well as with $K_{M}$ equal to $10^{-4}$ (corresponding to zero metabolism). The resulting uptake and desaturation curves are shown in Figure 2. The experimental data from a previous study (Teisinger et al., 1952; Teisinger and Fiserova-Bergerova, 1955) are also given in Figure 2. It is evident that the uptake and desaturation curves coincide with the experimental data only if $K_{M}$ is equal to $3 \cdot 2$.

In order to estimate the contribution of the individual compartments and the effect of metabolism on the total uptake, the currents (uptake or desaturation rates) passing through the individual branches of the network and the voltages (levels of saturation) on the capacitors VRG, MG, and FG were computed. The results are shown in Figures 3 and 4.

The effect of exposure duration on redistribution was studied. Exposure times of $\mathbf{3 0}$ minutes, one hour, two hours, five hours, and eight hours were simulated. Desaturation curves for these exposure times are shown in Figure 5.

In order to simulate industrial exposure, thecumulation of benzene in the body was computed for eight hours of exposure, five days per week, for six consecutive weeks. The uptake and desaturation curves are given in Figure 6. The broken line simulates the hypothetical condition that metabolism does not occur. In Figure 7 the saturation level of different compartments is demonstrated. It was calculated that six working weeks of exposure were needed to reach the steady state.

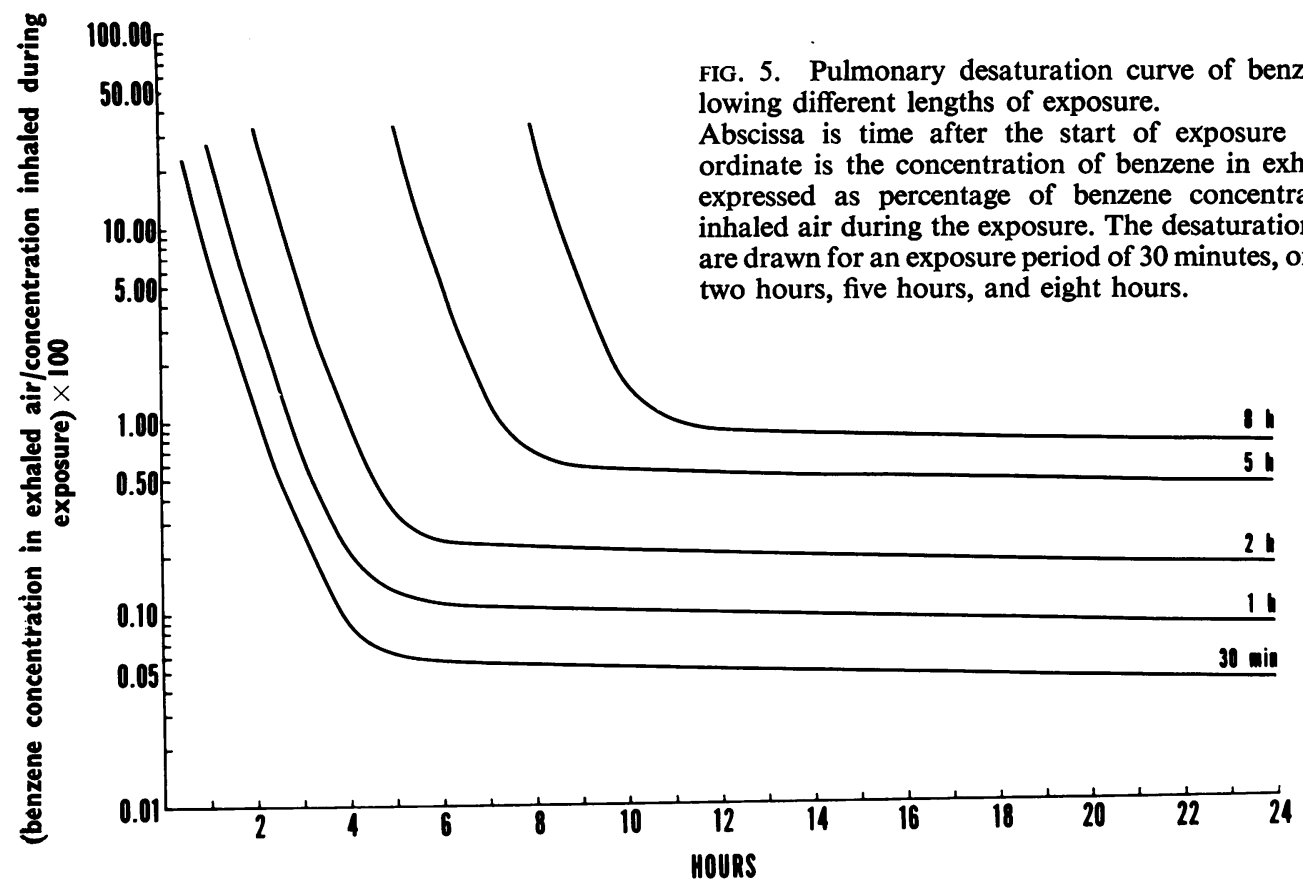

FIG. 5. Pulmonary desaturation curve of benzene following different lengths of exposure.

Abscissa is time after the start of exposure (hours); ordinate is the concentration of benzene in exhaled air inhaled air during the exposure. The desaturation curves are drawn for an exposure period of $\mathbf{3 0}$ minutes, one hour, two hours, five hours, and eight hours. 


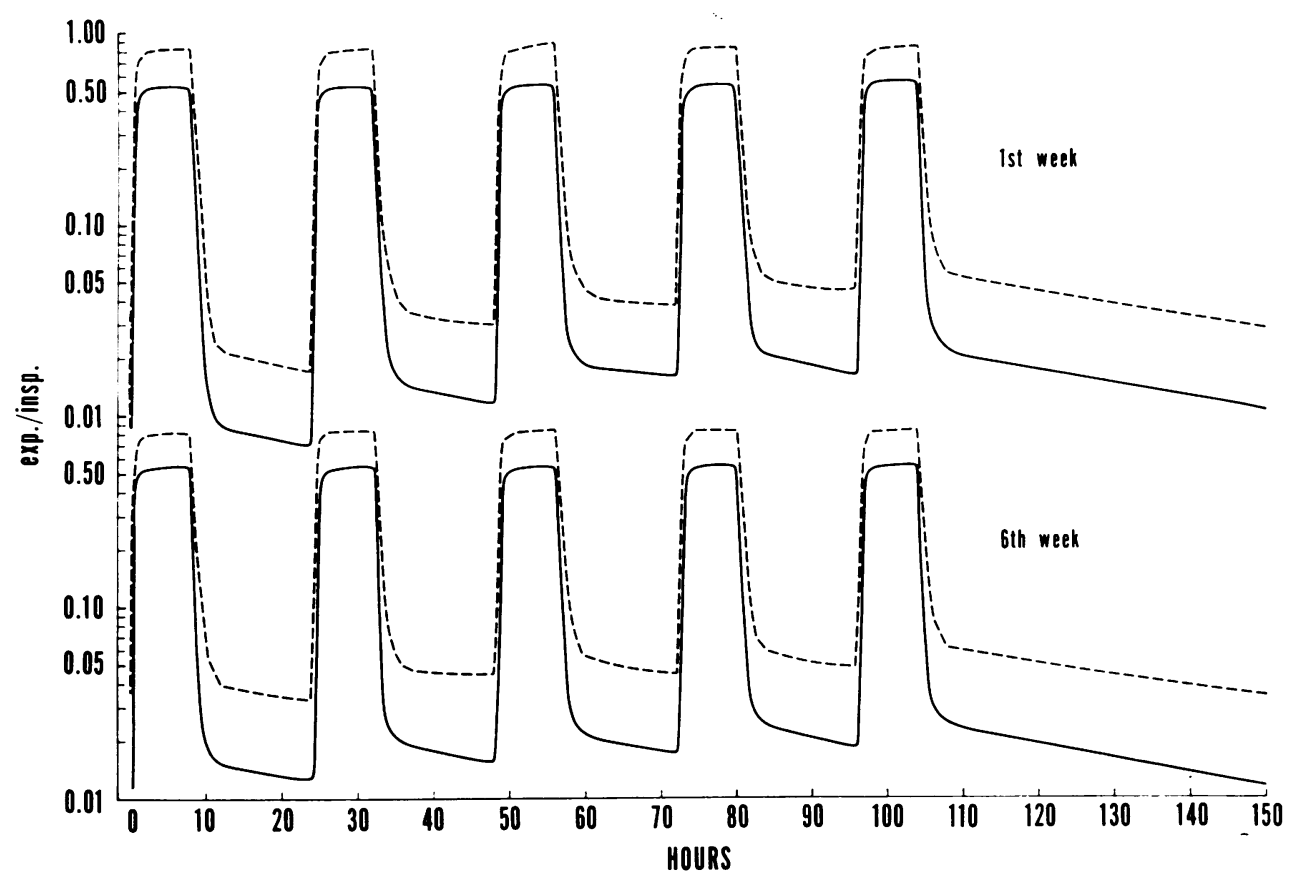

FIG. 6. Benzene concentration in exhaled air (exp) during the first and sixth weeks of chronic exposure. Exposure to a constant concentration of benzene (insp) eight hours per day on five consecutive days per week; unbroken line includes metabolism; broken line presumes zero metabolism.
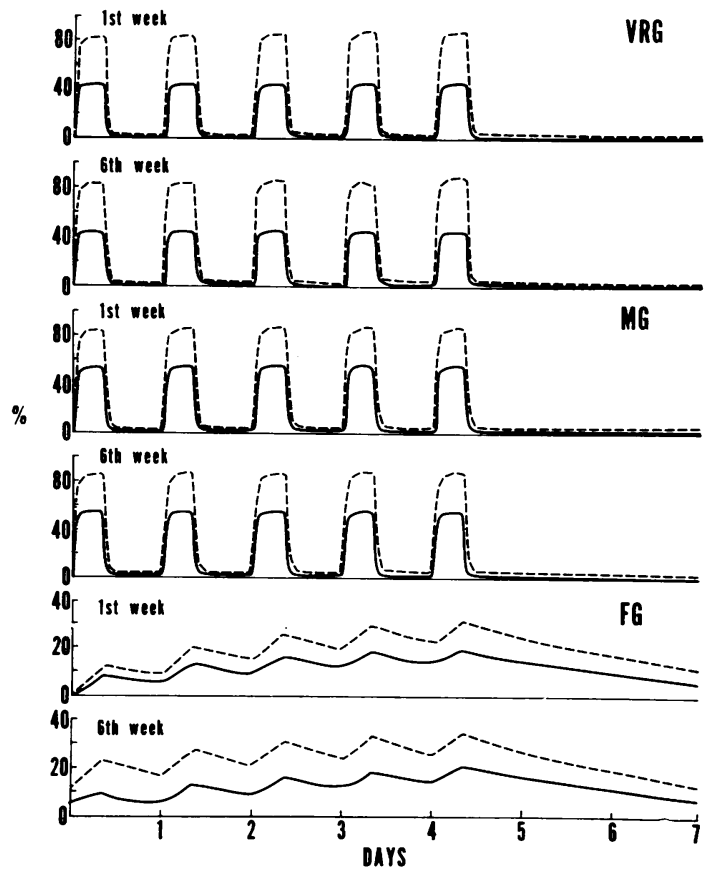

\section{Discussion}

Industrial hygienists attempt to monitor the exposure of workers to noxious compounds by measuring the concentrations exhaled or excreted in the original form or as metabolites in urine. Their most difficult problem is to evaluate the effect of exposure duration, and especially the effect of the time interval between the end of exposure and the collection of the exhaled air sample. The principal advantage of modelling the uptake, distribution, and excretion of drugs by an electrical network is the availability of a large body of knowledge for the solution of such networks. The mathematical theory used in this paper was chosen because it is not subject to the accumulation of errors frequently encountered when differential equations are solved for long periods of time. This is one of the reasons why a digital computer was preferred to an

FIG. 7. Benzene levels in pharmacokinetic compartments during the first and sixth weeks of chronic exposure. Exposure to constant concentration of benzene eight hours per day on five consecutive days per week: unbroken line includes metabolism; broken line presumes zero metabolism. 
analogue computer. It also enables easy calculation of 'steady state' situations, as occur in industrial exposure, without actually calculating the response during the transition time. Programming for a digital computer enables practically instant checking for different situations, and predictions can be made which are otherwise not possible, as for instance in the case of industrial exposure.

Metabolism plays a very significant role in uptake, distribution, and excretion of solvents (FiserovaBergerova, 1973) in any computation of uptake and desaturation curves. The electrical network simulating the curves in this study is similar to that of Mapleson (1963a), but an additional resistor connected in parallel to the capacitance VRG represents the metabolic shunt (Fiserova-Bergerova and Cettl, 1972). This study shows that the experimental data fit the computed curves. Metabolism increases uptake but depresses the concentrations of solvents in tissues, as well as depressing pulmonary desaturation (Figs. 3 and 4), since the metabolic output competes with pulmonary desaturation.

Uptake by the VRG compartments is very rapid at the beginning of exposure due to the great blood flow to tissues of this compartment. Because of the relatively small capacity of this compartment to absorb benzene, it would be saturated to $80 \%$ of capacity in two hours if no metabolism occurs (Figs 3 and 4), and the uptake by VRG would be negligible during further exposure. Because of metabolic output, the benzene uptake by the VRG compartment is increased, and it continues during the whole period of exposure, replacing the metabolized benzene. The newly arrived benzene is immediately subjected to metabolism, and therefore the benzene concentrations in tissues never reach such high levels as they would if metabolism did not occur (Fig. 4). The apparent high uptake by the VRG tissues, which accounts for metabolism, continues even during the desaturation period, by taking a fraction of drug released from other compartments.

Uptake by the MG compartment is also large at the beginning of exposure, but the uptake rate drops rapidly. It takes approximately two hours for uptake to become negligible under rest conditions. Without metabolism the MG compartment would be saturated to $80 \%$ of capacity (metabolism depresses the extent of saturation to $52 \%$ ). When exposure to benzene lasts longer than two hours, the partial pressure of solvent in the MG compartments is equilibrated with blood, and desaturation from the MG compartment begins immediately after the end of exposure (Figs 3 and 4). A significant amount of benzene is washed out within two hours. If the exposure is shorter than two hours, the partial pressure in the MG compartment is smaller than in blood, and the muscles continue in uptake for a moment during the desaturation period until the partial pressures are equilibrated. It is expected that physical stress, which increases the blood supply to the MG compartment, will accelerate the uptake and desaturation of this compartment.

Uptake by the FG compartment is very slow and is determined by the slow perfusion of fat. The time constant is large and the compartment is never saturated during industrial exposure. When exposure ends, benzene in the body is redistributed. While benzene from the VRG and MG compartments is being washed out, the FG compartment continues in uptake for a certain period until the partial pressures are equilibrated. The shorter the exposure, the greater is the redistribution. Redistribution would be much greater if metabolism did not occur. The washout of benzene from the FG compartment is a very slow process.

The exposure duration plays an important role in benzene redistribution. If exposure lasts 30 minutes, only VRG tissues are saturated, so that MG and FG compartments continue in saturation after the end of exposure. The concentration in the exhaled air during the entire desaturation depends on the concentration of benzene in inhaled air during the exposure, as well as on the duration of the exposure. On the other hand, if the exposure lasts two hours or longer, only the partial pressure in the FG compartment fails to reach equilibrium with blood. Immediately after the end of exposure, the concentration in exhaled air is approximately $32 \%$ of exposure concentration (Fig. 5). Benzene concentration in exhaled air drops rapidly during the first three postexposure hours. During this period the duration of exposure does not have any significant effect on exhalation. The duration of exposure plays an important role in desaturation, beginning five hours after the end of exposure. Benzene exhaled in this period is washed out mainly from the FG compartment. As the transport rate to the FG compartment during exposure is very slow, because of the low blood perfusion of these tissues, the saturation level of this compartment is proportional to the duration of exposure. The plot of concentration of benzene in exhaled air 24 hours after the start of exposure, $c_{24}$ (before the next work shift), against the duration of exposure, $t$ (in hours), is a straight line described by the equation

$$
c_{24}=0.875 \times 10^{-3} \times c_{i r,} \times t
$$

where $c_{\text {in }}$ is the concentration of benzene in the air during the exposure period.

What has been said so far is valid for only one acute exposure. In industrial conditions exposure is repetitive. As demonstrated in Figs 6 and 7, the time intervals between shifts are not long enough for complete washout of benzene from the body. This results in the cumulation of benzene. Each exposure increases the benzene level in the FG compartment. During exposure, as well as during desaturation, the 
concentration of benzene in exhaled air increases with the cumulation of benzene in fat. The most striking cumulative effect is seen in air samples collected five hours or more after the end of exposure. This is reflected in practice in the following way. The concentration of benzene in exhaled air in samples collected on Tuesday morning are one-third of that collected on Friday or Saturday morning during the first week of exposure. The concentrations of benzene in exhaled air are more nearly equal when the steady state is reached in the sixth week of exposure (Fig. 6).

From the computation of the model care is recommended in the interpretation of samples collected shortly after the end of the work shift, as the concentration in exhaled air drops rapidly with each minute after the end of exposure. If later collected samples are analysed (for example, samples collected before the next work shift), the duration of the previous exposure has to be considered, as was shown experimentally and recommended by Sherwood (1973). The curves in Fig. 5 or in equation (2) can be used as guidelines.

This study was supported by USPHS grant GMO-1714 and the National Research Council of Canada grant A 7398 .

\section{References}

Conney, A. H. (1972). Environmental factors influencing drug metabolism. In Fundamentals of Drug Metabolism and Drug Disposition, edited by B. N. La Du, H. G. Mandel and E. L. Way, pp. 253-265. Williams and Wilkins Company, Baltimore.

Eger, E. I. (1963). Mathematical model of uptake and distribution. In Uptake and Distribution of Anesthetic Agents, edited by E. M. Papper and R. J. Kitz, pp. 72-103. McGraw-Hill, New York.

Fiserova-Bergerova, V. (1973). Simulation of uptake, distribution, metabolism, and excretion of lipid soluble solvents in man. Proceedings of the Third Conference on Environmental Toxicology, AMRL-TR-72-130 Air-Space Medical Research Laboratory, Wright-Patterson Air Force Base, Ohio.

_ and Cettl, L. (1972). Electric analogue for uptake metabolism and excretion of benzene in man. Pracovni Lékarstvi, 24, 56-61.

Kozáková, H. (1955). Determination of partition coefficients between air, water and oil for benzene. Pracovni Lékarstvi, 7, 150-152.

Mapleson, W. W. (1963a). Quantitative prediction of anesthetic concentrations. In Uptake and Distribution of Anesthetic Agents, edited by E. M. Papper and R. J. Kitz, pp. 104-119. McGraw-Hill, New York.

- (1963b). An electric analogue for uptake and exchange of inert gases and other agents. Journal of Applied Physiology, 18, 197-204.

Schwarz, R. and Friedland, B. (1965). Linear Systems. McGraw-Hill, New York.

Severinghaus, J. W. (1963). Role of lung factors. In Uptake and Distribution of Anesthetic Agents, edited by E. M. Papper and R. J. Kitz, pp. 59-71. McGraw-Hill, New York.

Sherwood, R. J. (1973). Comparative methods of monitoring of benzene exposure. Proceedings of the Third Conference on Environmental Toxicology, AMRL-TR-72-130, AirSpace Medical Research Laboratory, Wright-Patterson Air Force Base, Ohio.
Teisinger, J. and Fiserova-Bergerova, V. (1955). Valeur comparée de la détermination des sulfates et du phénol contenus dans l'urine pour l'évaluation de la concentration du benzène dans l'air. Archives des Maladies Professionelles, 16, 221-232.

- _ - and Kudrna, J. (1952). The metabolism of benzene in man. Pracovni Lékarstvi, 4, 175-188.

— and Soucek, B. (1952). Significance of metabolism of toxic gases for their absorption and elimination by man. Casopis Lékarů Ceskjych, 91, 1372-1375.

Uptake and Distribution of Anesthetic Agents, (1963). Edited by E. M. Papper and R. J. Kitz. McGraw-Hill, New York.

\section{APPENDIX}

\section{Mathematical solution}

The mathematical description of the simulating network uses the Laplace transform. The reciprocal values of all resistors $G_{1}=1 / R_{\mathrm{i}}$ are used. A matrix equation describes the network:

$$
(\mathbf{G}+s \mathbf{C}) \mathbf{V}=\mathbf{J}
$$

where $G_{\mathrm{i}}$ are transportation rates of noxious vapours

$$
\begin{aligned}
G & =\left[\begin{array}{cccc}
G_{1}+G_{2}+G_{3}+G_{4} & -G_{2} & -G_{3} & -G_{4} \\
-G_{2} & G_{2}+G_{\mathrm{x}} & 0 & 0 \\
-G_{3} & 0 & G_{3} & 0 \\
-G_{4} & 0 & 0 & G_{4}
\end{array}\right] \\
G_{1} & =\text { pulmonary ventilation, } G_{2},,_{3},{ }_{4}=F_{1} \times \lambda \text { bl/air } \\
G_{\mathrm{x}} & =\frac{1}{\mathrm{R}_{\mathrm{x}}} .
\end{aligned}
$$$$
C=\left[\begin{array}{cccc}
C_{1} & 0 & 0 & 0 \\
0 & C_{2} & 0 & 0 \\
0 & 0 & C_{3} & 0 \\
0 & 0 & 0 & C_{4}
\end{array}\right] \begin{aligned}
& \text { The values of } C_{1} \text { represent the } \\
& \text { capacity of the tissues to } \\
& \text { retain noxious vapours }\left(C_{1}=\right. \\
& \begin{array}{l}
\text { volume of the compartment } \\
\times \lambda \text { tiss/air). }
\end{array}
\end{aligned}
$$$$
V=\left[\begin{array}{l}
V_{1} \\
V_{2} \\
V_{3} \\
V_{4}
\end{array}\right] \begin{aligned}
& \text { Voltages at the points with corres- } \\
& \text { ponding numbers (see Fig. 1) represent } \\
& \text { saturation levels of tissues by noxious } \\
& \text { vapours. }
\end{aligned}
$$

$\left[\begin{array}{c}Z G_{1} \\ 0\end{array}\right] \begin{aligned} & \text { Current sources where } Z=E \text { for the } \\ & \text { charging period (concentration of }\end{aligned}$ $\left.J=\left[\begin{array}{c}Z G_{1} \\ 0 \\ 0\end{array}\right] \begin{array}{l}\text { charging period (concentration of } \\ \text { noxious vapours in inhaled or alveolar }\end{array}\right]$ air during the uptake) and $Z=0$ for the discharging period (desaturation).

Because the operator ' $s$ ' in (1) is equivalent to differentiation, we can write

$$
\mathbf{G V}+\mathbf{C} \frac{\mathrm{d}}{\mathrm{dt}} \mathbf{V}=\mathbf{J}
$$

and

$$
\frac{\mathrm{d}}{\mathrm{dt}} \mathbf{V}=-\mathbf{C}^{-1} \mathbf{G V}+\mathbf{C}^{-1} \mathbf{J}=\mathbf{A V}+\mathbf{U}
$$

The solution of this equation can be found on any digital computer by a routine for integration of a system of linear differential equations. While such integration is easy and programmes are readily available, the results become unreliable when solutions are required for very long periods of time, such as for chronic uptake-desaturation encountered in industrial exposure. Recognizing that input $\mathbf{U}=\mathbf{C}^{-1} \mathbf{J}$ in (2) will be constant over long 
periods of time, the above set of differential equations can be transformed into a system of difference equations.

Let the input $U$ be allowed to change only at some multiple of a basic time interval $T$, and denote at the $n$th such instant its value by $\mathrm{U}(n)$, which corresponds to $\mathrm{U}(n T)$. Let the system be observed only at the time points $t=0, T, 2 T, \ldots n T$, and let $\mathrm{V}(n)$ represent the various voltages. Then equations (2) can be transformed to

$$
\mathbf{V}(n+1)=\hat{\mathbf{A}} \mathbf{V}(n)+\mathbf{M U}(n)
$$

where

$$
\begin{gathered}
\hat{\mathbf{A}}=e^{\mathbf{A} T} \\
\mathbf{M}=\int_{0}^{\mathbf{T}} e^{\mathbf{A T}} \mathrm{dt}
\end{gathered}
$$

The advantage of doing this is that the operations indicated in (4) and (5) are performed only once, and (3) involves very simple operations requiring no integration. For instance, periodic industrial exposure for eight hours a day, five days a week, can be calculated by only 21 evaluations of (3).

Many methods exist for determining $e^{\mathbf{A} t}$, but the observation of the system is desirable at short time intervals. This would require the computation of the exponential matrix for many values of $T$. It is thus preferable to find the analytical form,

$$
e^{\mathbf{A} t}=\sum B_{\mathbf{i}} e^{p_{i} t}
$$

where $B_{i}$ are matrices and $p_{i}$ denote various eigenvalues of $A$. The easiest way to find $B_{1}$ is to use the Laplace transform again by noting that

$$
e^{\mathbf{A} t}=\mathscr{L}^{-1}\left[(s \mathbf{I}-\mathbf{A})^{-1}\right]
$$

where I denotes the identity matrix. The expression $(s \mathbf{I}-\mathbf{A})^{-1}$ is found as a function of $s$ by the Leverrier algorithm. The determinant of $(s \mathbf{I}-\mathbf{A})$ is also found in the process, and its zero gives the eigenvalues $p_{i}$, and the residues of $(s I-\mathbf{A})^{-1}$ at these eigenvalues represent the $B_{i}$.

Once $e^{\mathbf{A}^{t}}$ has been found in analytical form, $\hat{\mathbf{A}}$ and $\mathbf{M}$ are given for any $T$ as

$$
\begin{gathered}
\hat{\mathbf{A}}=\sum \mathbf{B}_{\mathrm{i}} e^{p_{i} T} \\
\mathbf{M}=\sum \mathbf{B}_{\mathrm{i}} \frac{1}{p_{\mathrm{i}}}\left(e^{p_{i} T}-1\right)
\end{gathered}
$$

The procedure is described in Schwarz and Friedland (1965). The equations were programmed in Fortran IV. The IBM 360/75 or Univac 1106 computer was used for the solutions.

Received for publication March 21, 1973 Accepted for publication June 14, 1973 